\title{
Macrophages in trigeminal ganglion contribute to ectopic mechanical hypersensitivity following inferior alveolar nerve injury in rats
}

Dulguun Batbold', Masamichi Shinoda ${ }^{2 *}$ D, Kuniya Honda ${ }^{2}$, Akihiko Furukawa ${ }^{3}$, Momoko Koizumi $^{4}$, Ryuta Akasaka ${ }^{3}$, Satoshi Yamaguchi ${ }^{1}$ and Koichi Iwata ${ }^{2}$

\begin{abstract}
Background: Accidental mandibular nerve injury may occur during tooth extraction or implant procedures, causing ectopic orofacial pain. The exact mechanisms underlying ectopic orofacial pain following mandibular nerve injury is still unknown. Here, we investigated the role of macrophages and tumor necrosis factor alpha (TNFa) in the trigeminal ganglion (TG) in ectopic orofacial pain following inferior alveolar nerve transection (IANX).

Methods: IANX was performed and the mechanical head-withdrawal threshold (MHWT) in the whisker pad skin ipsilateral to IANX was measured for 15 days. Expression of Iba1 in the TG was examined on day 3 after IANX, and the MHWT in the whisker pad skin ipsilateral to IANX was measured following successive intra-ganglion administration of the macrophage depletion agent liposomal clodronate Clophosome-A (LCCA). TNFa expression in the TG and the MHWT in the whisker pad skin ipsilateral to IANX following successive intra-ganglion administration of the TNFa blocker etanercept were measured on day 3 after IANX, and tumor necrosis factor receptor-1 (TNFR1) immunoreactive (IR) cells in the TG were analyzed immunohistochemically on day 3.

Results: The MHWT in the whisker pad skin was significantly decreased for 15 days, and the number of Iba1-IR cells was significantly increased in the TG on day 3 after IANX. Successive intra-ganglion administration of the macrophage depletion agent LCCA significantly reduced the increased number of Iba1-IR cells in the TG and reversed the IANX-induced decrease in MHWT in the whisker pad skin. TNFa expression was increased in the TG on day 3 after IANX and was reduced following successive intra-ganglion administration of the TNFa inhibitor etanercept. The decreased MHWT was also recovered by etanercept administration, and TNFR1-IR cells in the TG were increased on day 3 following IANX.
\end{abstract}

Conclusions: These findings suggest that signaling cascades resulting from the production of TNFa by infiltrated macrophages in the TG contributes to the development of ectopic mechanical allodynia in whisker pad skin following IANX.

Keywords: Trigeminal ganglion, Tumor necrosis factor alpha, Macrophage, Ectopic orofacial pain, Inferior alveolar nerve transection, Mechanical allodynia

\footnotetext{
* Correspondence: shinoda.masamichi@nihon-u.ac.jp

${ }^{2}$ Department of Physiology, School of Dentistry, Nihon University, 1-8-13

Kandasurugadai, Chiyoda-ku, Tokyo 101-8310, Japan

Full list of author information is available at the end of the article
} 


\section{Background}

There are several causes of orofacial neuropathic pain, including accidental nerve injury or compression of the trigeminal nerve during dental treatments such as dental implant surgery and tooth extractions or facial trauma [1-3]. The orofacial pain may occasionally spread to intact orofacial regions, and clinicians struggle to alleviate the symptoms of ectopic orofacial pain. However, the detailed mechanisms of ectopic neuropathic pain are not fully understood.

Previous studies have indicated that pathological changes in the intra-trigeminal ganglion (TG) signaling mechanisms following trigeminal nerve injury are involved in the development of ectopic orofacial neuropathic pain. For example, nitric oxide released from the injured TG neurons and satellite glial activation via connexin 43 signaling modulates the excitability of intact TG neurons following inferior alveolar nerve injury (IANX) [4, 5]. Recently, immune cells that are infiltrated following peripheral nerve injury have been shown to play an important and diverse role in pathological neuronal changes. Sciatic nerve transection induces macrophage proliferation around axotomized dorsal root ganglion neurons and contributes to the regeneration of transected afferent nerve fibers [6-8]. Furthermore, sciatic nerve injury-induced macrophage invasion into the injured site and injury-induced mechanical and thermal hypersensitivity was abolished by macrophage depletion $[9,10]$. The infiltration of activated macrophages into the dorsal root ganglion results in the development of behavioral hypersensitivity in rats which induced chemotherapy-induced peripheral neuropathy [11].

Tumor necrosis factor alpha (TNF $\alpha)$ is well known as a proinflammatory cytokine involved in the development and maintenance of neuropathic pain. Increased TNF $\alpha$ was observed in the injured region in a model of sciatic nerve injury-induced mechanical and thermal hyperalgesia, and inhibition of TNF $\alpha$ synthesis abolished the hyperalgesia [12]. Activated macrophages are known to produce and release TNF $\alpha$ [13]. These results suggest that TNF $\alpha$ production by infiltrating macrophages may be involved in pain hypersensitivity following peripheral nerve injury.

Therefore, we hypothesized that the proliferation of activated macrophages in the TG contributes to the development of orofacial ectopic neuropathic pain following inferior alveolar nerve transection (IANX). In this study, we examined the relation between mechanical allodynia following IANX with the infiltration of macrophages into the TG and elucidated their functional roles in the development of ectopic orofacial neuropathic pain.

\section{Methods}

\section{Animals}

We used male Sprague-Dawley rats $(n=101$, Japan SLC, Shizuoka, Japan) weighing 170-260 g. All rats were housed individually in transparent polycarbonate cages (length $48 \mathrm{~cm}$; width $26.5 \mathrm{~cm}$; height $21 \mathrm{~cm}$ ) with paper shavings as bedding. The room temperature was controlled $\left(23{ }^{\circ} \mathrm{C}\right)$ with a $12 \mathrm{~h}$ light/dark cycle (lights on at 7:00, off at 19:00), and animals were allowed ad libitum access to food and water. All experiments were performed according to the National Institutes of Health Guide for the Care and Use of Laboratory Animals and the guidelines of the International Association for the Study of Pain [14]. This study was approved by the Animal Experimentation Committee at Nihon University (AP16D010).

\section{Inferior alveolar nerve transection}

IANX was performed as described previously under deep anesthesia with intraperitoneal (i.p.) butorphanol (2.5 mg/kg, Meiji Seika Pharma, Tokyo, Japan), medetomidine $(0.375 \mathrm{mg} / \mathrm{kg}$, Zenoaq, Fukushima, Japan), and midazolam (2.0 mg/kg, Sandoz, Tokyo, Japan) [15]. Briefly, a small incision was made in the skin of the left cheek, and the masseter muscle was dissected to expose the mandibular bone surface. The surface of the mandible was scraped using a low-speed dental drill bur to expose the inferior alveolar nerve. The exposed inferior alveolar nerve was gently pulled, transected, and replaced into the mandibular canal. In the control group, the skin incision, muscle dissection, and bone scraping without IANX were performed (sham operation). The dissected muscle and skin incision were sutured with 6-0 silk.

\section{Measuring mechanical sensitivity of the whisker pad skin}

Rats were trained daily for 5 to 7 days to protrude their snout from a cage that had a small fenestration and to withdraw their snout freely following application of mechanical stimuli to the whisker pad skin by von Frey filaments (Touch-Test Sensory Evaluator, North Coast Medical, Morgan Hill, CA) as described previously [4]. After stabilizing the mechanical head-withdrawal threshold (MHWT), IANX or sham operation was performed and followed by behavioral testing under the same conditions every other day for 15 days. The MHWT was determined as the lowest intensity that evoked headwithdrawal responses to five stimuli (duration $1 \mathrm{~s}$ ). The behavioral testing was conducted under blinded conditions.

Intra-TG administration of Clophosome-A and etanercept Under deep anesthesia with i.p. butorphanol $(2.5 \mathrm{mg} / \mathrm{kg})$, medetomidine $(0.375 \mathrm{mg} / \mathrm{kg})$, and midazolam $(2.0 \mathrm{mg} / \mathrm{kg})$, 
a head of rat was placed in a stereotaxic instrument for guidance $[4,16]$. The skull was exposed and a small hole ( $1 \mathrm{~mm}$ in diameter) was drilled above the location of the TG $(2.8 \mathrm{~mm}$ anterior from the posterior fontanelle, $2.7 \mathrm{~mm}$ lateral to the sagittal suture) ipsilateral to the location of IANX or sham operation. A guide cannula was extended in the TG (9 mm below the skull surface) and was anchored to the skull bone with dental cement and three stainless-steel screws. To confirm the correct positioning of the tip of the cannula, a trocar was inserted into the cannula as an electrode and multi-unit activities induced by mechanical stimulation of the whisker pad skin were conformed. After implantation of the cannula, rats were allowed to recover for 5 days before performing the IANX or sham operation. Under light anesthesia with $2 \%$ isoflurane in oxygen, liposomal clodronate Clophosome-A (LCCA, $6 \mu \mathrm{l}, 7 \mathrm{mg} / \mathrm{mL}$, product code: F70101CA, FormuMax Scientific, Sunnyvale, CA, USA) or plain control liposomes for LCCA (Cont-LCCA, $6 \mu \mathrm{l}$, $20 \mathrm{mM}$, product code: F70101-A, FormuMax Scientific) were administered into the TG through the guide cannula prior to operation and on days 1 to 4 following IANX or sham operation. LCCA has been shown to be effective in successfully depleting proliferated and resident macrophages [17]. Etanercept (1 $\mu$ g; Pfizer, New York, NY, USA) was dissolved in physiological saline or vehicle alone (physiological saline) and administered $(6 \mu \mathrm{l})$ into the TG through the guide cannula under light anesthesia with $2 \%$ isoflurane in oxygen prior to operation and on days 1 to 4 following IANX or sham operation. The LCCA and etanercept administration were performed immediately after the measurement of the MHWT of whisker pad skin. The MHWT of the whisker pad skin was measured before operation and every other day for 5 days after IANX or sham operation.

Moreover, rats were allowed to recover after implantation of the cannula for 5 days. Under light anesthesia with $2 \%$ isoflurane in oxygen, $2 \mu \mathrm{l}$ of $1,1^{\prime}$-dioctadecyl3,3,3',3-tetramethylindocarbocyanine methanesulfonate (DiI, Thermo Fisher Scientific, Waltham, MA) was administered into the TG through the guide cannula. On day 3 after the administration, rats were deeply anesthetized with the above-described solution and transcardially perfused with saline followed by a fixative containing $4 \%$ paraformaldehyde in $0.1 \mathrm{M}$ phosphate buffer (PB; $\mathrm{pH}$ 7.4). After the perfusion, TGs were removed and immersed in a fixative containing $4 \%$ paraformaldehyde for more than $4 \mathrm{~h}$ at $4{ }^{\circ} \mathrm{C}$ then transferred to $0.01 \mathrm{M}$ phosphate-buffered saline (PBS) for $12 \mathrm{~h}$ for cryoprotection. TG from naive rat was also processed in a similar manner. Next, the tissues were embedded in Tissue-Tek (Sakura Finetechnical, Tokyo, Japan) and were cut in $12-\mu \mathrm{m}$-thick sections in a horizontal plane along the long axis.

\section{Immunohistochemistry}

Under deep anesthesia with i.p. butorphanol $(2.5 \mathrm{mg} / \mathrm{kg})$, medetomidine $(0.375 \mathrm{mg} / \mathrm{kg})$, and midazolam $(2.0 \mathrm{mg} / \mathrm{kg})$, a retrograde labeling tracer, FluoroGold $(7 \mu \mathrm{l}$ dissolved in saline; FG; 4\% hydroxystilbamidine; Fluorochrome, Denver, CO, USA), was injected into the skin of the left whisker pad ipsilateral to IANX using a 30-gauge needle before performing the IANX or sham operation. On day 3 , rats were deeply anesthetized with the above-described solution and transcardially perfused with saline followed by a fixative containing $4 \%$ paraformaldehyde in $0.1 \mathrm{M}$ phosphate buffer (PB; pH 7.4). After the perfusion, TGs were removed and immersed in the same fixative for more than $4 \mathrm{~h}$ at $4{ }^{\circ} \mathrm{C}$ then transferred to $0.01 \mathrm{M}$ PBS containing $20 \%$ sucrose for $12 \mathrm{~h}$ for cryoprotection. Next, the tissues were embedded in Tissue-Tek and stored at $-20{ }^{\circ} \mathrm{C}$ until cryosectioning. Then, the ganglion tissues were cut in $12-\mu \mathrm{m}$-thick sections in a horizontal plane along the long axis. Every 10th section (five sections per rat) was thawmounted on a MAS-GP slide glass (Matsunami, Osaka, Japan) and dried overnight at room temperature. The five sections were used for immunohistological analysis. After rinsing with $0.01 \mathrm{M}$ PBS three times for 5 min each, sections were incubated in mouse anti-Iba1 polyclonal antibody (1:200; Abcam, Cambridge, MA, USA) and rabbit anti-TNF $\alpha$ monoclonal antibody (1:200; Abcam) or rabbit anti-glial fibrillary acidic protein (GFAP) polyclonal antibody (1:500; cat.No. ab7260, Abcam) or rabbit anti-F4/80 monoclonal antibody (1:500; cat.No. ab111101, Abcam), or anti-tumor necrosis factor receptor-1 (TNFR1) monoclonal antibody (1:50; Santa Cruz, Santa Cruz, CA, USA), diluted in $0.01 \mathrm{M}$ PBS containing 4\% normal donkey serum (Merck, Darmstadt, Germany) in 0.3\% Triton $\mathrm{X}-100$ (Merck) overnight at $4{ }^{\circ} \mathrm{C}$. After rinsing with $0.01 \mathrm{M}$ PBS, the sections were incubated in Alexa Fluor 568 anti-rabbit IgG (1:200; Thermo Fisher Scientific) and/ or Alexa Fluor 488 anti-mouse IgG (1:200; Thermo Fisher Scientific) in $0.01 \mathrm{M}$ PBS for $2 \mathrm{~h}$ at room temperature. After rinsing with $0.01 \mathrm{M} \mathrm{PBS}$, sections were coverslipped in PermaFluor (Thermo Fisher Scientific). The sections were observed using a BZ-9000 system (Keyence, Osaka, Japan). Iba1-IR and TNF $\alpha$-IR cells in the TGs of the ophthalmic-maxillary division (V1-V2) and mandibular division (V3) were analyzed. The mean relative area (density) of Iba1-IR or TNF $\alpha$-IR cells were calculated by using a computer-assisted imaging analysis system (ImageJ, NIH, Bethesda, MD, USA). The mean relative area (density) of F4/80- and Iba1-IR area was calculated by using a computer-assisted imaging analysis system (BZ-X analyzer, Keyence, Osaka, Japan). All immunohistochemical measurements were conducted under blinded conditions, performed by observers who were unaware of which treatment the animals received. Using the same conditions, no specific labeling was observed in the 
absence of primary antibody. Measurement of the area occupied by the immuno-products was made in a region selected identically from V1/V2 and V3 in the TG.

\section{Western blotting}

On day 3 following IANX or sham operation, the rats were perfused through the aorta with physiological saline under deep anesthesia as described above. Immediately, the TG was removed and homogenized in ice-cold lysis buffer (137 mM NaCl; $20 \mathrm{mM}$ Tris-HCL, $\mathrm{pH}$ 8.0; 1\% NP40; $10 \%$ glycerol; $1 \mathrm{mM}$ phenylmethylsulfonyl fluoride; $10 \mu \mathrm{g} / \mathrm{ml}$ aprotinin; $1 \mathrm{~g} / \mathrm{ml}$ leupeptin; $0.05 \mathrm{mM}$ sodium vanadate). The homogenate was centrifuged at $15,000 \mathrm{rpm}$ for $10 \mathrm{~min}$ at $4{ }^{\circ} \mathrm{C}$, and the protein concentration of the resulting supernatants was determined using a protein assay kit (Bio-Rad, Hercules, CA, USA). The supernatants were heat-denatured in Laemmli sample buffer solution (Bio-Rad), and $30 \mu \mathrm{g}$ of each protein sample was subjected to SDS-PAGE using a $10 \%$ acrylamide gel. The samples were transferred to a polyvinylidene difluoride membrane (Trans-Blot Turbo Transfer Pack, Bio-Rad) using Trans-Blot Turbo (Bio-Rad). The membrane was rinsed with Tris-buffered saline mixed with $0.1 \%$ Tween 20 (TBST) and incubated in 3\% bovine serum albumin (BSA; Bovogen, Essendon, Australia). The membrane was then incubated with rabbit anti-TNF $\alpha$ polyclonal antibody (1:200; diluted in TBST and 3\% BSA, Abcam) or rabbit anti-Iba1 monoclonal antibody (1:1000; diluted in TBST and 3\% BSA, cat.No. ab178847, Abcam) overnight at $4{ }^{\circ} \mathrm{C}$. Next, it was incubated with horseradish peroxidase-conjugated donkey anti-rabbit antibody (Cell Signaling, Danvers, MA, USA) for $2 \mathrm{~h}$ at room temperature. Antibody binding was detected using Western Lightning ELC Pro (PerkinElmer, Waltham, MA, USA). Band intensity was analyzed using a ChemiDoc MP system (Bio-Rad) and normalized to $\beta$-actin on blots re-probed with the anti- $\beta$-actin antibody (1:200; Santa Cruz) after removing bound protein using a stripping reagent (Thermo Scientific).

\section{Statistical analysis}

Data were expressed as mean \pm standard error. Statistical analyses were performed by Student's $t$ test, one-way analysis of variance (ANOVA) with Tukey's multiplecomparison test, and two-way ANOVA with repeated measures followed by Bonferroni's multiple-comparison test where appropriate. A value of $p<0.05$ was defined as statistically significant.

\section{Results}

Mechanical allodynia and Iba1 expression in TG following IANX

The MHWT of the whisker pad skin ipsilateral to IANX was significantly decreased on day 1 following IANX, and this decrease persisted until day 15 when compared with sham-operated animals (Fig. 1a). On day 3 following IANX, Iba1-IR cells were observed in V1/V2 FG-labeled neurons, as well as in V3, in which FG-labeled neurons were absent (Fig. 1b, c). The relative area of Iba1-IR cells in V1/V2 and V3 in TG ipsilateral to IANX was significantly increased on day 3 following IANX compared to that of the sham-operated group (Fig. 1d). The Iba1 protein level was increased in TG ipsilateral to IANX on day 3 following IANX compared to shamoperated animals (Fig. 1e).

\section{Effect of intra-TG LCCA administration on mechanical allodynia and Iba1 expression following IANX}

Primarily, it was confirmed that drug administration into the TG through the guide cannula was accurate (Fig. 2a). Daily administration of LCCA into the TG prevented the development and progress of mechanical allodynia in the whisker pad skin ipsilateral to IANX from days 1 to 5 following IANX (Fig. 2b). On day 3 after IANX, Iba1-IR cells in V1/V2, in which FG-labeled neurons were abundant, were observed in the LCCA- and Cont-LCCA-treated groups (Fig. 2c). Moreover, the relative area of Iba1-IR cells in the LCCA-treated group was significantly lower compared to the group treated with Cont-LCCA (Fig. 2d). Before intra-TG LCCA and Cont-LCCA administration, it was confirmed that the guide cannula implantation did not change the mechanical sensitivity (data not shown).

\section{TNFa expression in TG following IANX}

TNF $\alpha$ was expressed in activated Iba1-IR cells presented with morphological characteristics such as the bigger cell bodies and thicker ramifications and in activated GFAP-IR cells in V1/V2 on day 3 in the IANX group rather than sham group (Fig. 3a). F4/80 immunoreactivity was observed in the Iba1-IR cells in V1/V2 on day 3 in IANX or sham groups, and the relative F4/80-IR area in the Iba1-IR area was decreased in V1/V2 ipsilateral to IANX compared to that of the sham-operated group (Fig. 3b). The relative area of TNFa immuno-products increased in V1/ V2 ipsilateral to IANX compared to that of the shamoperated group (Fig. 3c, d). The TNF $\alpha$ protein level was also increased in TG ipsilateral to IANX on day 3 following IANX compared to naive and sham-operated animals (Fig. 3e). Daily LCCA administration significantly reduced the relative area of TNF $\alpha$ immuno-products in V1/V2 in TG on day 3 following IANX compared to the ContLCCA-treated IANX group or sham group (Fig. 4a, b).

\section{Effect of intra-TG etanercept administration in TG on mechanical allodynia following IANX}

Daily administration of etanercept into the ipsilateral TG prevented mechanical hypersensitivity in whisker 

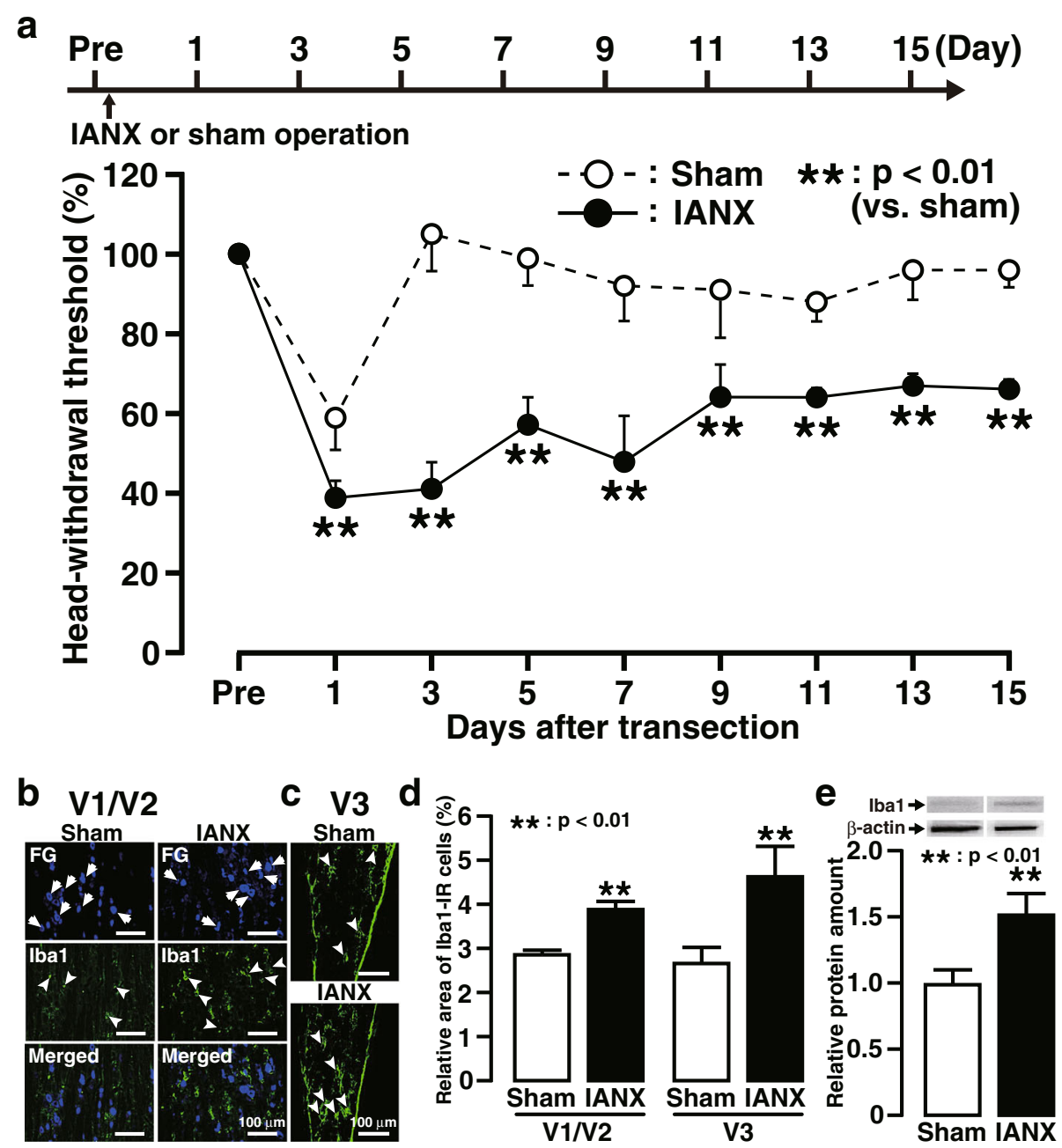

Fig. 1 Mechanical allodynia and Iba1 expression in the TG following IANX. a The timing of IANX or sham operation (upper) and the changes in MHWT of the ipsilateral whisker pad skin for 15 days following IANX or sham operation (lower). The MHWT was normalized to the pre-IANX MHWT (100\%). Error bars indicate SEM. ${ }^{* *} p<0.01$ vs. sham ( $n=6$ in each; two-way ANOVA with repeated measures followed by Bonferroni's multiple-comparison test). Iba1-IR cells in V1/N2 (b) and V3 (c) in the ipsilateral TG on day 3 following IANX or sham operation. Arrows indicate FG-labeled neurons. Arrowheads indicate Iba1-IR cells. $\mathbf{d}$ The relative area of Iba1-IR cells in V1/N2 and V3 in TG on day 3 following IANX and sham operation. Error bars indicate SEM. ** $p<0.01$ vs. sham. ( $n=5$ in each, Student's $t$ test). e The relative amount of Iba1 protein in TG on day 3 following IANX or sham operation. Error bars indicate SEM. ${ }^{*} p<0.05$ vs. sham ( $n=10$ in each, Student's $t$ test)

pad skin ipsilateral to IANX from days 1 to 5 (Fig. 5a). Immunostaining was performed to examine TNFR1 expression in the TG on day 3 following IANX. TNFR1 expression was observed in FG-labeled neurons, and the mean percentage of FG-labeled TNFR1-IR neurons in TG ipsilateral to IANX was significantly increased compared to the sham-operated group (Fig. 5b).

\section{Discussion}

Traumatic injury to the inferior alveolar nerve, which can occur during extraction of an impacted mandibular third molar or mandibular plastic surgery, occasionally causes ectopic pain hypersensitivity in widespread orofacial areas such as the forehead and maxillary regions [1].
In this study, IANX produced long-lasting mechanical allodynia in ipsilateral whisker pad skin, as reported in our previous studies $[4,15,18]$. The long-lasting mechanical allodynia observed in the IANX model mimics ectopic pain hypersensitivity in patients with a history of traumatic injury to the inferior alveolar nerve, indicating that this model may be useful for research into the mechanisms of ectopic orofacial neuropathic pain.

Iba1 expression is known to be widely used as a specific macrophage marker [19]. IANX induced extensive macrophage infiltration in not only $\mathrm{V} 3$ but also in V1/V2 and increased Iba1 expression in the ipsilateral TG. Moreover, the infiltrated macrophages showed the bigger cell bodies and thicker ramifications, which 

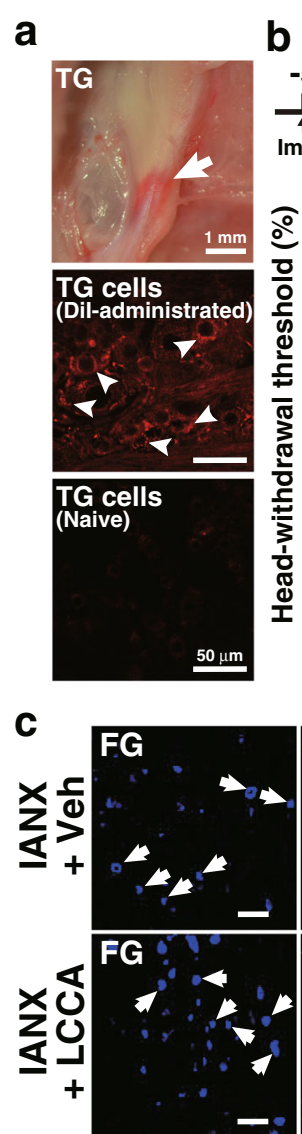

b
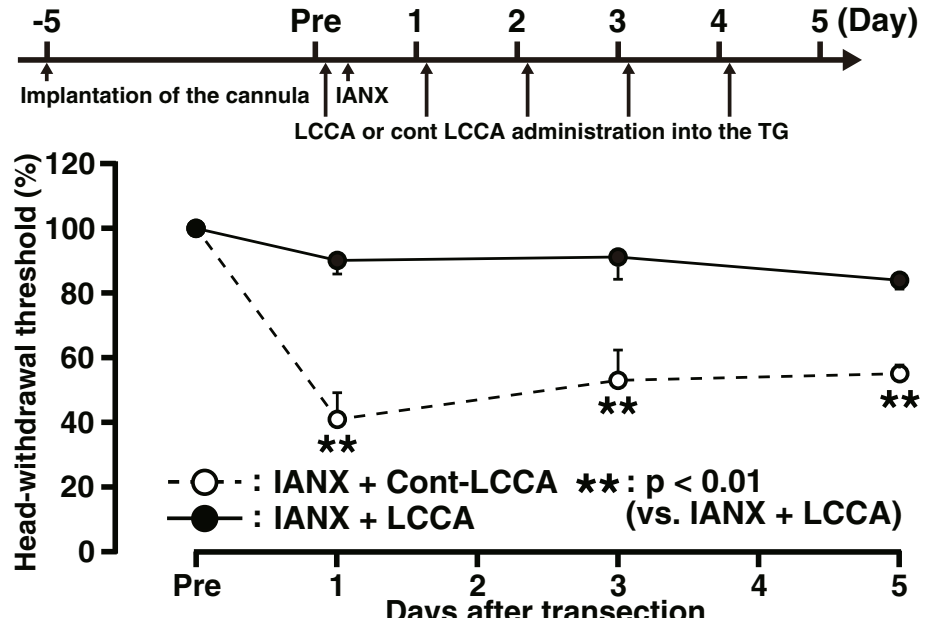

- O- : IANX + Cont-LCCA $* *: p<0.01$
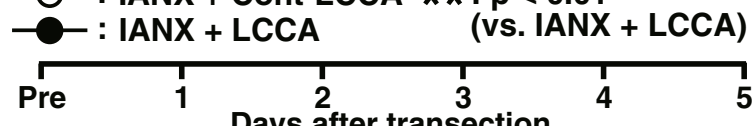

Days after transection
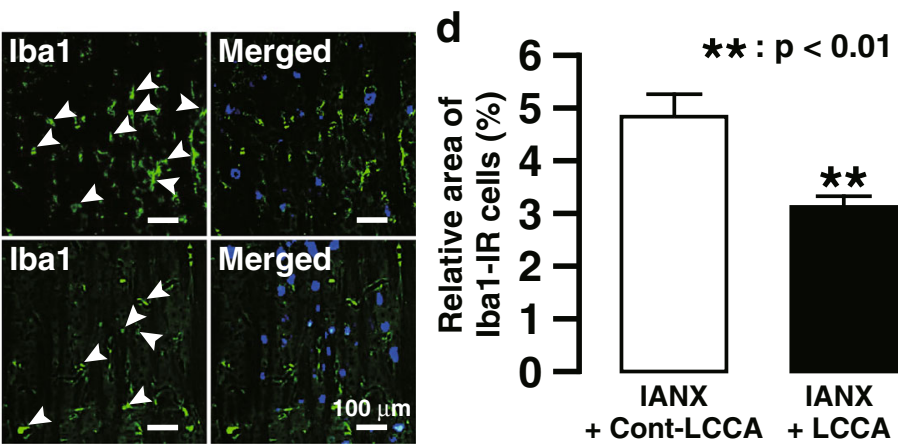

Fig. 2 Effect of intra-TG LCCA administration on mechanical allodynia and Iba1 expression following IANX. a Photograph (upper) and photomicrograph (middle) of TG on day 3 following Dil administration into the TG through the guide cannula and photomicrograph (lower) of TG in naive rat. Arrows indicate Dil labeling. $\mathbf{b}$ The timing of the cannula implantation, LCCA, or Cont-LCCA administration into the TG, IANX, or sham operation (upper) and the changes in MHWT of the ipsilateral whisker pad skin following daily intra-TG LCCA or Cont-LCCA administration following IANX (lower). The MHWT was normalized to the pre-IANX MHWT (100\%). Arrows indicate the timing of intra-TG LCCA or Cont-LCCA administration. Error bars indicate SEM. ${ }^{* *} p<0.01$ vs. sham ( $n=6$ in each; two-way ANOVA with repeated measures followed by Bonferroni's multiple-comparison test). c Iba1-IR cells and FGlabeled TG neurons in V1N2 on day 3 following intra-TG LCCA or Cont-LCCA administration in IANX rats. Arrows indicate FG-labeled neurons. Arrowheads indicate Iba1-IR cells. $\mathbf{d}$ The relative area of Iba1-IR cells in V1N2 in TG on day 3 following intra-TG LCCA or Cont-LCCA administration in IANX rats. Error bars indicate SEM. ${ }^{* *} p<0.01$ vs. sham. ( $n=5$ in each, Student's $t$ test)

indicates their activation [20]. Previous studies have shown that nerve injury increases resident and proliferated macrophages in the sensory ganglion [21-23]. The chemokine $\mathrm{C}-\mathrm{C}$ motif ligand 2 (CCL2), also referred to as monocyte chemoattractant protein 1 , is released from injured neurons and activates the $\mathrm{C}-\mathrm{C}$ chemokine receptor type 2 (CCR2) in macrophages, which induces macrophage activation and proliferation in the dorsal root ganglion (DRG) following peripheral nerve injury $[8,24]$. Moreover, CCL2 hyper-expression and macrophage infiltration in the DRG, induced by peripheral nerve injury, is reduced in Toll-like receptor 2 (TLR2) knock-out mice [25]. Based on these results, we hypothesize that the extensive infiltration of activated macrophages in the TG ipsilateral to IANX observed here was induced by CCL2 released from injured neurons via TLR2 signaling. F4/80 expression is known to be used as tissue-resident macrophage marker [26, 27]. The relative F4/80-IR area in the Iba1-IR area in TG was decreased following IANX in this study, suggesting that the proliferation of the activated macrophages in TG ipsilateral to IANX is mainly caused by exogenous macrophage infiltration into the TG.

The proliferation of activated macrophages was observed in the TG ipsilateral to IANX, and these activated macrophages expressed TNF $\alpha$. IANX significantly increased TNF $\alpha$ expression in the ipsilateral TG, and macrophage depletion in the TG significantly suppressed the increase in TNF $\alpha$ expression. Macrophages are believed to be the major source of TNF $\alpha$ in the sensory ganglion following peripheral nerve injury [28, 29]. Intracellular signaling cascades such as p38 mitogenactivated protein kinase (MAPK) and extracellular signal-regulated kinase (ERK) cascades result in 

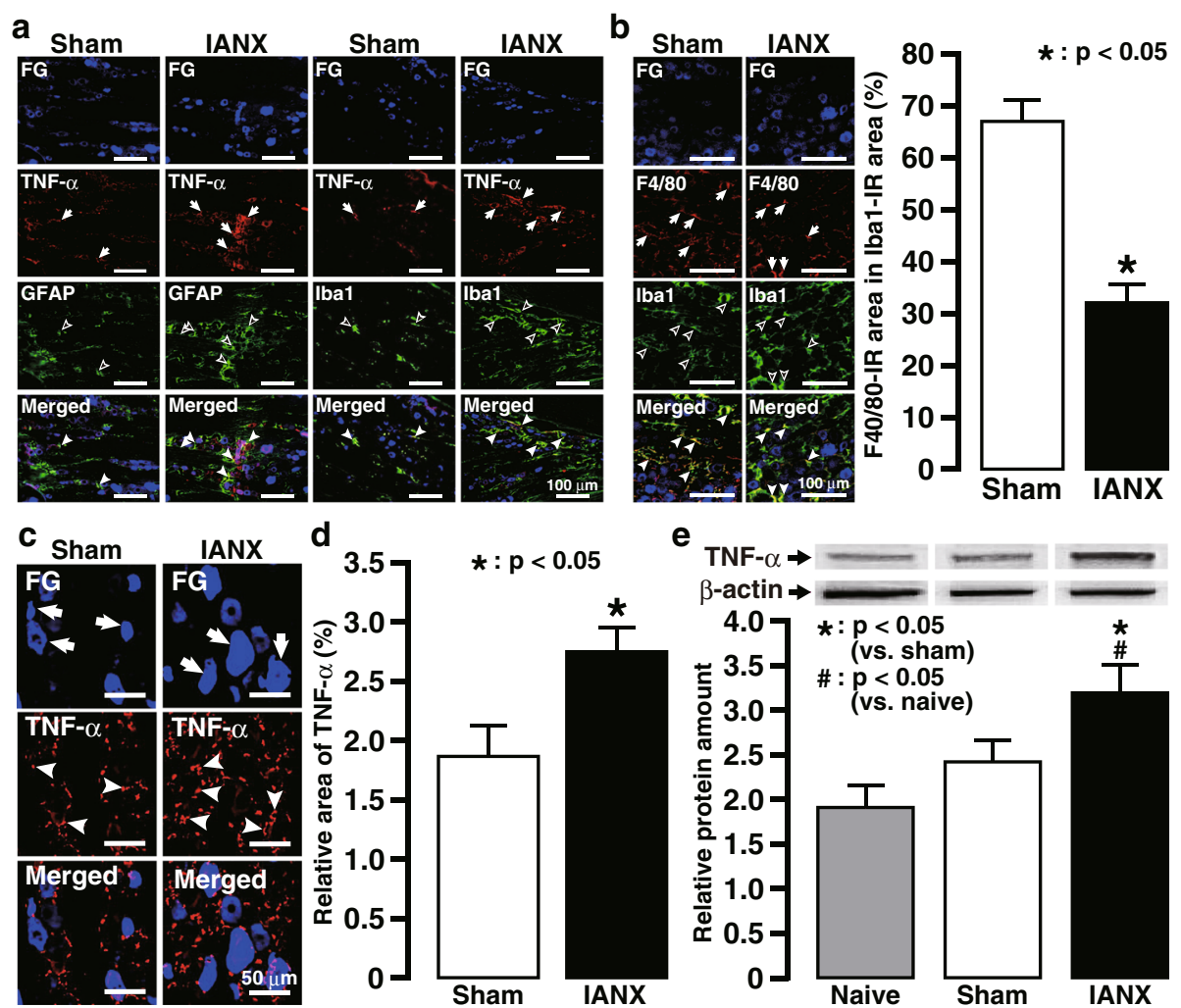

Fig. 3 TNFa expression in TG following IANX. a TNFa expression in the Iba1-IR cells or the GFAP-IR cells in ipsilateral TG on day 3 following IANX or sham operation. Arrows indicate TNFa-IR cells, open arrowheads indicate GFAP-IR cells or Iba1-IR cells, and close arrowheads indicate TNFa- and GFAPIR cells or TNFa- and Iba1-IR cells. b F4/80 expression in the Iba1-IR cells in ipsilateral TG on day 3 following IANX or sham operation (left). Arrows indicate F4/80 expression in Iba1-IR cells, open arrowheads indicate Iba1-IR cells, and close arrowheads indicate F4/80- and Iba1-IR cells. The relative area of F4/80 immuno-products in Iba1-IR cells in the TG on day 3 following IANX or sham operation (right). Error bars indicate SEM. ${ }^{* * *} p<0.001$ vs. sham. ( $n=5$ in each, Student's $t$ test). c TNFa expression in ipsilateral TG on day 3 following IANX or sham operation. Arrows indicate FG-labeled neurons. Arrowheads indicate TNFa immuno-products. $\mathbf{d}$ The relative area of TNFa immuno-products in the TG on day 3 following IANX or sham operation. Error bars indicate SEM. ${ }^{*} p<0.05$ vs. sham ( $n=5$ in each, Student's $t$ test). e The relative amount of TNFa protein in TG in naive animals or on day 3 following IANX or sham operation. Error bars indicate SEM. ${ }^{*} p<0.05$ vs. sham. \#p $<0.05$ vs. naive ( $n=5$ in each, one-way ANOVA with Tukey's multiple-comparison test)
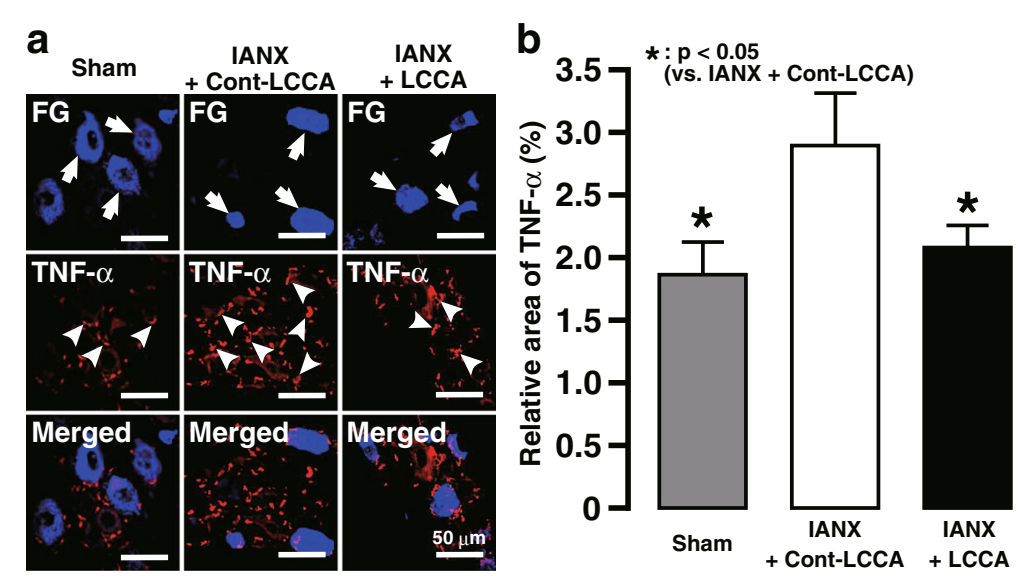

Fig. 4 Changes in TNFa expression in TG following LCCA administration following IANX. a TNFa expressed in V1N2 in the TG on day 3 following intra-TG LCCA or Cont-LCCA administration in IANX rats or in sham-operated rats. Arrows indicate FG-labeled neurons. Arrowheads indicate TNFa immunoproducts. $\mathbf{b}$ Changes in the relative area of TNF-a immuno-products in the TG on day 3 following intra-TG LCCA or Cont-LCCA administration in IANX rats or in sham-operated rats. Error bars indicate SEM. ${ }^{*} p<0.05$ vs. Cont-LCCA. ( $n=5$ in each, one-way ANOVA with Tukey's multiple-comparison test) 


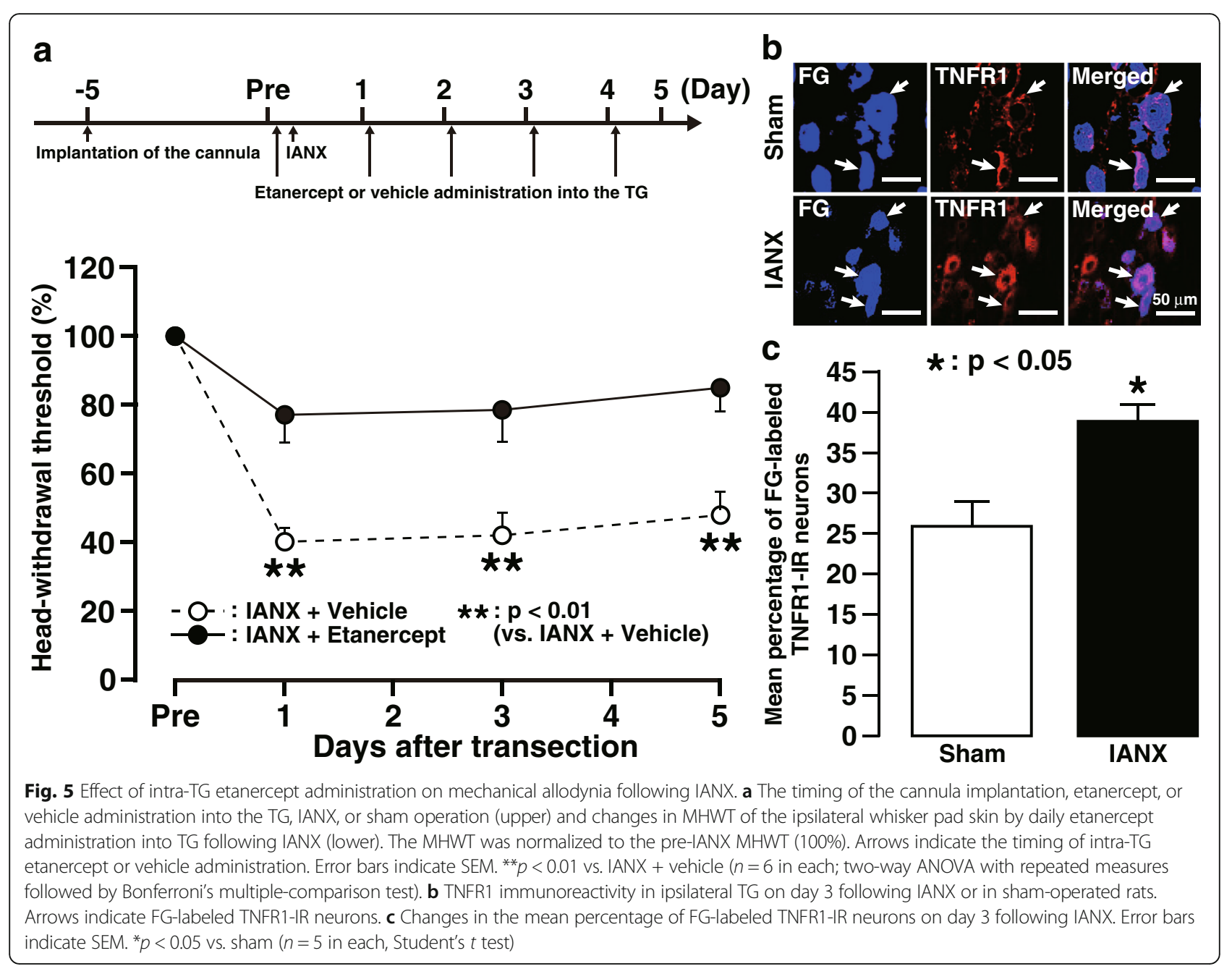

macrophage activation and TNF $\alpha$ release [30]. The expression of substance $\mathrm{P}$ (SP), which is a member of the tachykinin family and a major mediator of neuroimmunomodulation, was found to be enhanced in the sensory ganglion neurons, and SP was released in the early stages after peripheral nerve injury [31]. Neurokinin 1 receptor which is activated by SP is present in macrophage, SP modulates diverse functions of macrophages, and SP signaling activates the ERK $1 / 2$ and p38 MAPK cascades [32-34]. Moreover, the activation of the p38 MAPK cascade and the ensuing TNF $\alpha$ production are induced in macrophages infiltrating into the nerveinjured site [35]. Previous studies have reported that TNF $\alpha$ levels in DRG increased following peripheral nerve injury [36, 37]. Taken together, these results suggest that SP released from TG neurons is transmitted to macrophages, resulting in macrophage activation and TNF $\alpha$ release from activated macrophages via the ERK $1 / 2$ and p38 MAPK signaling cascades. Furthermore, GFAP immunoreactivity which is a marker of the activated satellite glial cells was observed in the TG ipsilateral to IANX, and these activated satellite glial cells also expressed TNF $\alpha$. Our previous report also indicates that activation of satellite glial cells in the TG was induced after IANX [4], suggesting that the activated satellite glial cells might be another source of TNF $\alpha$ in the TG after IANX. Further studies are needed.

In this study, TNFR1 expression in TG neurons innervating the whisker pad skin and the increase of TNF $\alpha$ in V1/V2 in the TG were observed following IANX. The continuous suppression of TNF $\alpha$ signaling and macrophage depletion in the TG prevented the development and progress of mechanical allodynia in the whisker pad skin ipsilateral to IANX. It was reported that intra-DRG administration of exogenous TNF $\alpha$ depolarizes DRG neurons and increases their neuronal excitability [38]. TNF $\alpha$ signaling in uninjured DRG neurons mediates the activation of voltage-gated sodium channels (Nav) in a dose-dependent manner by upregulating current densities of tetrodotoxin (TTX)-sensitive and TTX-resistant Nav 1.3 and Nav 1.8 in the neurons via p38 MAPK cascade following peripheral nerve injury [36, 39, 40]. 
TNF $\alpha$ also increases membrane potassium ion conductance in a non-voltage-gated fashion, resulting in the enhancement of neuronal excitability [41, 42]. We have previously reported that satellite glial cells are activated throughout the TG via gap junctions following IANX [4]. Adenosine triphosphate was released from sensory neurons following peripheral nerve injury, and TNF $\alpha$ was released from activated satellite glial cells through $\mathrm{P} \mathrm{X}_{7}$ signaling $[43,44]$. Based on the existing reports, this study suggests that TNF $\alpha$ released from proliferated macrophages or satellite glial cells binds to TNFR1 in TG neurons, and TNF $\alpha$-TNFR1 signaling might contribute to the enhancement of TG neuronal excitability, resulting in mechanical allodynia in the whisker pad skin following IANX, whereas IANX significantly increased TNF $\alpha$ expression in not only the activated macrophages but also in the satellite cells other than the activated macrophages in TG. Some reports indicated that peripheral nerve injury induced TNF $\alpha$ hyper-expression in the satellite cells in DRG, suggesting that TNF $\alpha$ released from the satellite cells following IANX also modulates mechanical sensitivity in the whisker pad skin which occurs as a result of TG neuronal excitability $[45,46]$.

TNFR1 expression in TG neurons innervating the whisker pad skin was significantly increased following IANX, and neutralizing TNFR1 attenuated thermal and mechanical allodynia following peripheral nerve injury [47]. Moreover, TNFR1 knockout mice do not develop mechanical allodynia induced by peripheral nerve injury [36]. Though these reports indicate that TNFR1 signaling plays a pivotal role in the development of mechanical allodynia and contributor to the enhancement of TNFR1 expression in TG neurons innervating the whisker pad skin following IANX, the detailed mechanism remains to be elucidated.

\section{Conclusions}

The signaling cascades induced by TNF $\alpha$ released from infiltrated macrophages in the TG contribute to the development of orofacial neuropathic ectopic pain following IANX. Therefore, TNF $\alpha$ signaling in the TG should be considered as one of the primary targets in the modulation and treatment of ectopic orofacial pain attributed to peripheral trigeminal nerve injury.

\footnotetext{
Abbreviations

ANOVA: Analysis of variance; BSA: Bovine serum albumin; CCL2: Chemokine C-C motif ligand 2; CCR2: C-C chemokine receptor type 2; Cont-LCCA: Plain control liposomes for LCCA; DRG: Dorsal root ganglion; ERK: Extracellular signal-regulated kinase; FG: FluoroGold; i.p.: Intraperitoneal; IANX: Inferior alveolar nerve transection; IR: Immunoreactive; LCCA: Liposomal clodronate Clophosome-A; MAPK: Mitogen-activated protein kinase; MHWT: Mechanical head-withdrawal threshold; Nav: Voltage-gated sodium channels; PB: Phosphate buffer; PBS: Phosphate-buffered saline; SP: Substance P; TBST: Tris-buffered saline mixed with 0.1\% Tween 20; TG: Trigeminal ganglion; TLR2: Toll-like receptor 2; TNFR1: Tumor necrosis factor receptor-1; TNFa: Tumor necrosis factor alpha; TTX: Tetrodotoxin; V1-V2: Ophthalmic-maxillary division; V3: Mandibular division
}

\section{Acknowledgements}

This study was supported in part by research grants from Sato and Uemura Funds from the Nihon University School of Dentistry, a grant from the Dental Research Center in Nihon University School of Dentistry, KAKENHI (Grant-in-Aid for Challenging Exploratory Research 15K15748), Nihon University Multidisciplinary Research Grant for (2016 and 2017), and MEXT-Supported Program for the Strategic Research Foundation at Private Universities 2013-2017.

\section{Availability of data and materials}

Almost all the data and materials supporting the conclusions of this study are presented in the manuscript. Alternatively, please contact corresponding author for requests of data.

\section{Authors' contributions}

All authors discussed the results and commented on the manuscript. DB, AF, $M K, R A$, and SY performed experiments and manuscript editing. MS and KI performed results analysis and wrote the manuscript. KH performed results analysis and generation of figures. MS was responsible for the study concept and design, results analysis, and manuscript writing. All authors read and approved the final manuscript.

\section{Ethics approval}

The experimental protocols including all the surgical procedures and animal usages conformed to the guidelines for the care and use of laboratory animals by the National Institutes of Health Guide for the Care and Use of Laboratory Animals and the guidelines of the International Association for the Study of Pain. This study was approved by the Animal Experimentation Committee at Nihon University (AP16D010).

\section{Consent for publication}

Not applicable.

\section{Competing interests}

The authors declare that they have no competing interests.

\section{Publisher's Note}

Springer Nature remains neutral with regard to jurisdictional claims in published maps and institutional affiliations.

\section{Author details}

'Department of Oral and Maxillofacial Surgery, Graduate School of Medical and Dental Sciences, Tokyo Medical and Dental University, 1-5-45 Yushima, Bunkyo-ku, Tokyo 113-8549, Japan. ${ }^{2}$ Department of Physiology, School of Dentistry, Nihon University, 1-8-13 Kandasurugadai, Chiyoda-ku, Tokyo 101-8310, Japan. ${ }^{3}$ Department of Clinical Medicine, School of Dentistry, Nihon University, 1-8-13 Kandasurugadai, Chiyoda-ku, Tokyo 101-8310, Japan. ${ }^{4}$ Department of Dentistry, Jikei University School of Medicine, 3-25-8 Nishi-Shimbashi, Minato-ku, Tokyo 105-8461, Japan.

Received: 23 August 2017 Accepted: 5 December 2017

Published online: 16 December 2017

\section{References}

1. Renton T, Yilmaz Z, Gaballah K. Evaluation of trigeminal nerve injuries in relation to third molar surgery in a prospective patient cohort. Recommendations for prevention. Int J Oral Maxillofac Surg. 2012;41:1509-18.

2. Khawaja N, Renton T. Case studies on implant removal influencing the resolution of inferior alveolar nerve injury. Br Dent J. 2009;206:365-70.

3. Lin CS, SY W, Huang HY, et al. Systematic review and meta-analysis on incidence of altered sensation of mandibular implant surgery. PLoS One. 2016;11:e0154082.

4. Kaji K, Shinoda M, Honda K, et al. Connexin 43 contributes to ectopic orofacial pain following inferior alveolar nerve injury. Mol Pain. 2016;12: doi:10.1177/1744806916633704

5. Sugiyama $T$, Shinoda $M$, Watase $T$, et al. Nitric oxide signaling contributes to ectopic orofacial neuropathic pain. J Dent Res. 2013:92:1113-7.

6. Gensel JC, Nakamura S, Guan Z, et al. Macrophages promote axon regeneration with concurrent neurotoxicity. J Neurosci. 2009:29:3956-68.

7. Kwon MJ, Kim J, Shin H, et al. Contribution of macrophages to enhanced regenerative capacity of dorsal root ganglia sensory neurons by conditioning injury. J Neurosci. 2013;33:15095-108. 
8. Kwon MJ, Shin HY, Cui Y, et al. CCL2 mediates neuron-macrophage interactions to drive proregenerative macrophage activation following preconditioning injury. J Neurosci. 2015;35:15934-47.

9. Liu T, van Rooijen N, Tracey DJ. Depletion of macrophages reduces axonal degeneration and hyperalgesia following nerve injury. Pain. 2000;86:25-32.

10. Kobayashi Y, Kiguchi N, Fukazawa Y, et al. Macrophage-T cell interactions mediate neuropathic pain through the glucocorticoid-induced tumor necrosis factor ligand system. J Biol Chem. 2015;290:12603-13.

11. Zhang H, Li Y, de Carvalho-Barbosa M, et al. Dorsal root ganglion infiltration by macrophages contributes to paclitaxel chemotherapy-induced peripheral neuropathy. J Pain. 2016;17:775-86.

12. George A, Marziniak M, Schafers $M$, et al. Thalidomide treatment in chronic constrictive neuropathy decreases endoneurial tumor necrosis factor-alpha, increases interleukin-10 and has long-term effects on spinal cord dorsal horn met-enkephalin. Pain. 2000;88:267-75.

13. Pang M, Yuan $Y$, Wang $D$, et al. Recombinant CC16 protein inhibits the production of pro-inflammatory cytokines via NF-kappaB and p38 MAPK pathways in LPS-activated RAW264.7 macrophages. Acta Biochim Biophys Sin Shanghai. 2017;49:435-43.

14. Zimmermann M. Ethical guidelines for investigations of experimental pain in conscious animals. Pain. 1983;16:109-10.

15. Iwata K, Imai T, Tsuboi Y, et al. Alteration of medullary dorsal horn neuronal activity following inferior alveolar nerve transection in rats. J Neurophysiol. 2001;86:2868-77.

16. Katagiri A, Shinoda M, Honda $K$, et al. Satellite glial cell P2Y12 receptor in the trigeminal ganglion is involved in lingual neuropathic pain mechanisms in rats. Mol Pain. 2012:8:23.

17. Van Rooijen N, Sanders A. Liposome mediated depletion of macrophages: mechanism of action, preparation of liposomes and applications. J Immunol Methods. 1994;174:83-93.

18. Okada-Ogawa A, Nakaya Y, Imamura Y, et al. Involvement of medullary GABAergic system in extraterritorial neuropathic pain mechanisms associated with inferior alveolar nerve transection. Exp Neurol. 2015;267:42-52.

19. Ohsawa $\mathrm{K}$, Imai $\mathrm{Y}$, Kanazawa $\mathrm{H}$, et al. Involvement of Iba1 in membrane ruffling and phagocytosis of macrophages/microglia. J Cell Sci. 2000;113(Pt 17):3073-84.

20. Harvey LD, Yin Y, Attarwala IY, et al. Administration of DHA reduces endoplasmic reticulum stress-associated inflammation and alters microglial or macrophage activation in traumatic brain injury. ASN Neuro. 2015;7(6): doi:10.1177/1759091415618969.

21. Donegan $M$, Kernisant $M$, Cua $C$, et al. Satellite glial cell proliferation in the trigeminal ganglia after chronic constriction injury of the infraorbital nerve. Glia. 2013;61:2000-8.

22. Komori T, Morikawa Y, Inada T, et al. Site-specific subtypes of macrophages recruited after peripheral nerve injury. Neuroreport. 2011;22:911-7.

23. Lu X, Richardson PM. Responses of macrophages in rat dorsal root ganglia following peripheral nerve injury. J Neurocytol. 1993;22:334-41.

24. Abbadie C, Lindia JA, Cumiskey AM, et al. Impaired neuropathic pain responses in mice lacking the chemokine receptor CCR2. Proc Natl Acad Sci U S A. 2003:100:7947-52

25. Kim D, You B, Lim H, et al. Toll-like receptor 2 contributes to chemokine gene expression and macrophage infiltration in the dorsal root ganglia after peripheral nerve injury. Mol Pain. 2011;7:74.

26. Mass E, Ballesteros I, Farlik M, et al. Specification of tissue-resident macrophages during organogenesis. Science. 2016;353(6304):aaf4238.

27. Hashimoto D, Chow A, Noizat C, et al. Tissue-resident macrophages selfmaintain locally throughout adult life with minimal contribution from circulating monocytes. Immunity. 2013;38:792-804.

28. Raghavendra V, Tanga F, Rutkowski MD, et al. Anti-hyperalgesic and morphine-sparing actions of propentofylline following peripheral nerve injury in rats: mechanistic implications of spinal glia and proinflammatory cytokines. Pain. 2003;104:655-64.

29. Wagner R, Myers RR. Endoneurial injection of TNF-alpha produces neuropathic pain behaviors. Neuroreport. 1996;7:2897-901.

30. Wang $C L, C Y$ L, Pi CC, et al. Extracellular polysaccharides produced by Ganoderma formosanum stimulate macrophage activation via multiple pattern-recognition receptors. BMC Complement Altern Med. 2012;12:119.

31. Fu C, Yin Z, Yu D, et al. Substance $P$ and calcitonin gene-related peptide expression in dorsal root ganglia in sciatic nerve injury rats. Neural Regen Res. 2013;8:3124-30.

32. Sun J, Ramnath RD, Zhi L, et al. Substance P enhances NF-kappaB transactivation and chemokine response in murine macrophages via ERK1/2 and p38 MAPK signaling pathways. Am J Physiol Cell Physiol. 2008;294: C1586-96.

33. Matsumoto K, Nakajima T, Sakai H, et al. Increased expression of 5-HT3 and NK 1 receptors in 5-fluorouracil-induced mucositis in mouse jejunum. Dig Dis Sci. 2013;58:3440-51.

34. Bardelli C, Gunella G, Varsaldi F, et al. Expression of functional NK1 receptors in human alveolar macrophages: superoxide anion production, cytokine release and involvement of NF-kappaB pathway. Br J Pharmacol. 2005;145:385-96.

35. Nomura H, Furuta A, Tanaka Y, et al. Forced retraction of spinal root injury enhances activation of p38 MAPK cascade in infiltrating macrophages. Neuropathology. 2005;25:37-47.

36. Chen X, Pang RP, Shen KF, et al. TNF-alpha enhances the currents of voltage gated sodium channels in uninjured dorsal root ganglion neurons following motor nerve injury. Exp Neurol. 2011;227:279-86.

37. Miao P, Madec K, Gong Y, et al. Axotomy-induced up-regulation of tumor necrosis factor-alpha in the dorsal root ganglia. Neurol Res. 2008;30:623-31.

38. Schafers $M$, Lee $D H$, Brors $D$, et al. Increased sensitivity of injured and adjacent uninjured rat primary sensory neurons to exogenous tumor necrosis factor-alpha after spinal nerve ligation. J Neurosci. 2003;23:3028-38.

39. He XH, Zang $Y$, Chen $X$, et al. TNF-alpha contributes to up-regulation of Nav1.3 and Nav1.8 in DRG neurons following motor fiber injury. Pain. 2010; 151:266-79.

40. Jin X, Gereau RW. Acute p38-mediated modulation of tetrodotoxin-resistant sodium channels in mouse sensory neurons by tumor necrosis factor-alpha. J Neurosci. 2006;26:246-55

41. Czeschik JC, Hagenacker T, Schafers M, et al. TNF-alpha differentially modulates ion channels of nociceptive neurons. Neurosci Lett. 2008;434:293-8.

42. Leo M, Argalski S, Schafers M, et al. Modulation of voltage-gated sodium channels by activation of tumor necrosis factor receptor-1 and receptor- 2 in small DRG neurons of rats. Mediat Inflamm. 2015;2015:124942.

43. Kanno T, Yaguchi T, Nishizaki T. Noradrenaline stimulates ATP release from DRG neurons by targeting beta(3) adrenoceptors as a factor of neuropathic pain. J Cell Physiol. 2010;224:345-51.

44. Zhang $X$, Chen $Y$, Wang $C$, et al. Neuronal somatic ATP release triggers neuron-satellite glial cell communication in dorsal root ganglia. Proc Natl Acad Sci U S A. 2007;104:9864-9.

45. Dubovy $\mathrm{P}$, Jancalek R, Klusakova I, et al. Intra- and extraneuronal changes of immunofluorescence staining for TNF-alpha and TNFR1 in the dorsal root ganglia of rat peripheral neuropathic pain models. Cell Mol Neurobiol. 2006; 26:1205-17.

46. Hatashita S, Sekiguchi M, Kobayashi H, et al. Contralateral neuropathic pain and neuropathology in dorsal root ganglion and spinal cord following hemilateral nerve injury in rats. Spine (Phila Pa 1976). 2008;33:1344-51.

47. Sommer C, Schmidt C, George A. Hyperalgesia in experimental neuropathy is dependent on the TNF receptor 1. Exp Neurol. 1998;151:138-42.

\section{Submit your next manuscript to BioMed Central and we will help you at every step:}

- We accept pre-submission inquiries

- Our selector tool helps you to find the most relevant journal

- We provide round the clock customer support

- Convenient online submission

- Thorough peer review

- Inclusion in PubMed and all major indexing services

- Maximum visibility for your research

Submit your manuscript at www.biomedcentral.com/submit 